\title{
Optic Neuritis Presenting With Altitudinal Visual Field Defect in a Neuromyelitis Optica Patient
}

\author{
Halil Onder ${ }^{\mathrm{a}, \mathrm{b}}$, Farid Khasiyev ${ }^{\mathrm{a}}$, Rana Karabudak ${ }^{\mathrm{a}}$
}

\begin{abstract}
Herein, we report a case with neuromyelitis optica (NMO) who presented with optic neuritis and visual field examination revealed superior altitudinal visual field defect. Via the presentation of this patient, we point out NMO as an alternative differential diagnosis in patients with altitudinal visual defect clinics. Based on this presentation and literature data, we discuss some hypotheses about pathogenesis of optic neuritis in NMO and underlying mechanisms of optic neuritis development presenting with altitudinal visual defect as well.
\end{abstract}

Keywords: Altitudinal visual defect; Optic neuritis; NMO; Neuroimaging

\section{Introduction}

Altitudinal visual field defect is classically believed to be highly characteristic of ischemic optic neuropathy and it is explained to occur due to occlusion of posterior ciliary artery [1]. Recently, other disorders such as neuromyelitis optica (NMO) has also been suggested to cause optic neuritis (ON) presenting with altitudinal field defect [2]. Herein, we illustrate clinical and neuroimaging findings of a patient presenting with $\mathrm{ON}$ in the form of altitudinal visual field defect who finally received the diagnosis of NMO. Via the detailed illustration of this patient and literature knowledge, we discuss the possible mechanisms behind altitudinal visual field defect in distinct etiologies other than ischemic optic neuropathy.

\section{Case Report}

A 31-year-old female was admitted to our center with com-

Manuscript submitted December 16, 2017, accepted December 27, 2017

aDepartment of Neurology, Hacettepe University Hospital, Sihhiye, Ankara, Turkey

${ }^{\mathrm{b} C}$ Corresponding Author: Halil Onder, Department of Neurology, Hacettepe University Hospital, Sihhiye, Ankara 06010, Turkey.

Email: halilnder@yahoo.com

doi: https://doi.org/10.14740/jnr463w plaints of vision impairment in the left eye that had started and progressed gradually in a 2-day period. Concurrently, the patient had began experiencing left periocular pain during eye movements. She had been smoking 1 packet cigarettes a day for 2 years and other medical history was unremarkable. Best corrected visual acuity was 20/30 in the left eye and color vision was evaluated as $1 / 6$. Direct pupillary light reflex was diminished in the left eye. Confrontational visual field exam suggested superior altitudinal vision defect in the left eye which was confirmed in automated perimetry (Fig. 1). Right eye examinations were found to be normal (visual acuity, pupillary light reflex, and visual field exam). Fundus examinations were bilateral normal. Brain MRI revealed normal brain parenchyma, but contrast enhancement in the intraorbital part of the left optic nerve was observed (Fig. 2a). Cervical MRI was unremarkable (Fig. 2b). At this point, features including the progressive course of symptoms, periocular pain and lack of vascular risk factors suggested rather the diagnosis of demyelinating optic neuropathy. Intravenous (IV) methylprednisolone was administered for 7 days. A dramatic recovery was achieved and low dosage alternate-day oral prednisolone $(8 \mathrm{mg}$ ) was continued for 1 month which resulted in a total recovery of vision acuity and visual field defect. Cerebrospinal fluid investigation results were unremarkable (normal IgG index, negative oligoclonal band, serology for infectious diseases, culture, and direct staining). Serum markers for vasculitic proceses were negative. Taken together, diagnosis of $\mathrm{ON}$ was made and timely outpatient follow-ups were planned without any further treatment suggestion. However, on 6 months of follow-up, she had an attack of paraparesis and numbness in bilateral upper limbs. Concurrently, cranial MRI was normal, but spinal cord MRI showed multiple, intermittent, central-medullary located lesions in cervicothoracic region and a longitudinally extensive lesion from T3 to T12 segment (Fig. 2c). Serum NMO IgG was positive. Hence, the patient was diagnosed with NMO, and after IV methylprednisolone treatment, oral azathioprine (150 mg daily) was started. Moderate improvement was achieved in the acute period. At the last follow-up, 6 months later, the patient had not experienced a new clinical attack and her neurological status was stable.

\section{Discussion}

ON, which can be defined as inflammation of the optic nerve due to various causes, is the most common optic neuropa- 


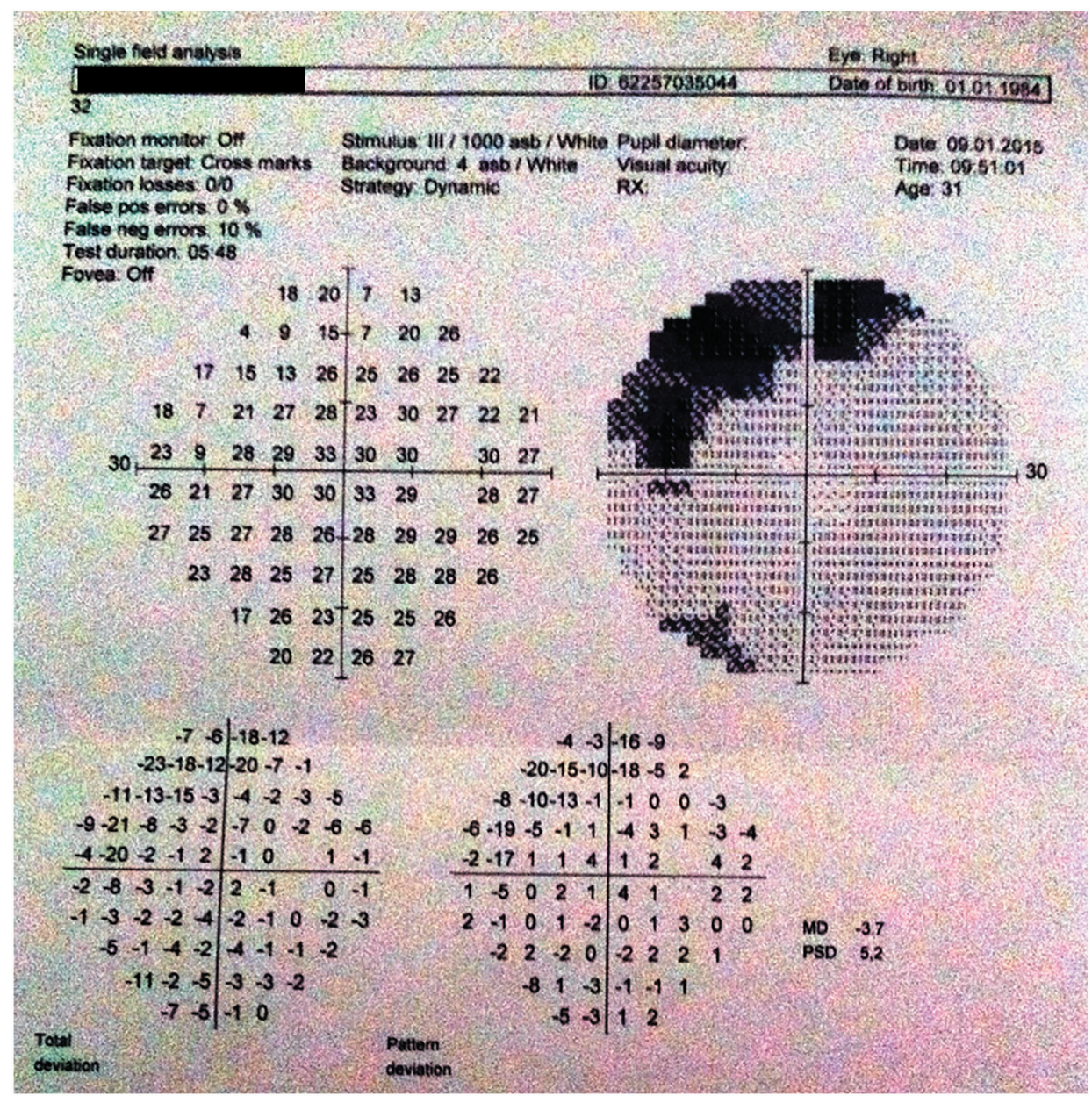

Figure 1. Automated perimetry revealed superior altitudinal visual defect in the left eye.

thy under 50 years among general ophthalmic practice [3]. Although the pathogenesis of $\mathrm{ON}$ is not fully understood, inflammatory processes induced by cytokines and other inflammatory mediators crossing blood brain barrier have been suggested as responsible mechanisms leading to myelin destruction, and additionally axonal degeneration [4]. Clinically, it usually manifests with visual loss, periocular pain and dyschromatopsia, and most commonly seen visual field defect is the type of diffuse depression and central or centrocecal scotoma. However, altitudinal visual defect is reported to be rather suggestive of anterior ischemic optic neuropathy which develops mainly due to the disruption in the posterior ciliary artery circulation [5]. On the other hand, in a study by Nakajiuma et al, altitudinal visual defect was also shown to be the most frequent non-central scotom pattern in NMO patients. Based on their results, they suggested possible NMO-IgG mediated ischemic injury as an explanation of $\mathrm{ON}$ development in NMO patients [2]. At this point, we would like to state that cranial MRI findings of our patient may add substantial perspectives to these arguments. Contrary to the hypothesis of Nakajima et al, our patient' s cranial MRI revealed thickening and contrast enhancement of intraorbital segment of left optic nerve which rather considered an under- lying inflammatory process other than ischemia. In addition, fundus examination was normal that was incompatible with ischemic optic neuropathy in which papilledema is stated as an essential finding in the early phase [6]. Taken together, we think that the manifestation of superior altitudinal vision defect might be rather primarily associated with an inflammatory process. Nonetheless, for our opinion, still a possible secondary perfusion defect that might have occurred due to the pressure rise related with inflammation of the anterior optic nerve cannot be ruled out likewise hypothesized by Hayreh [6].

In conclusion, we present a crucial illustration of $\mathrm{ON}$ in association with NMO which was in the form of altitudinal visual defect and we discuss the hypothesis of secondary ischemic process of posterior ciliary artery circulation [6]. For our opinion, this report may give crucial insights in respect of underlying mechanisms of $\mathrm{ON}$ in NMO. On the other hand, this may also give some views concerning underlying pathophysiology of ON presenting with altitudinal visual field defects. Nonetheless, future reports of large case studies including neuroimaging findings need to clarify these arguments. Finally, via the presentation of this case we again remark NMO as an alternative differential diagnosis in patients presenting 


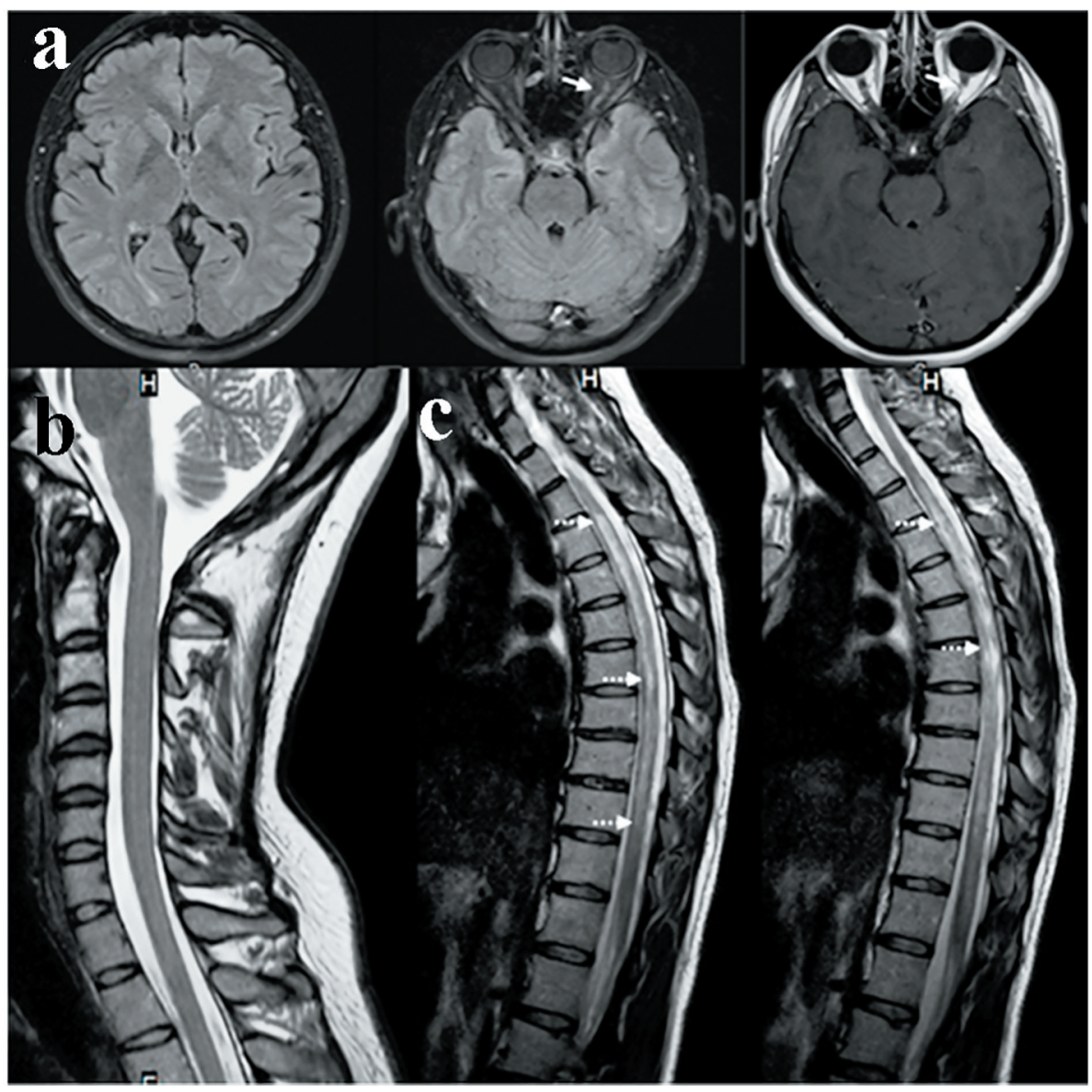

Figure 2. (a) Cranial MRI at first admission showing thickening and contrast enhancement of intraorbital segment of left optic nerve (arrows). (b) Normal findings in cervical MRI (at first attack). (c) Spinal MRI at second attack showing longitudinal cervicothoracic extensive cord lesion (intermittent arrows).

with altitudinal visual defects.

\section{Consent}

Informed consent was obtained from the subject.

\section{Conflict of Interest}

There is not any conflict of interest.

\section{References}

1. Gerling J, Meyer JH, Kommerell G. Visual field defects in optic neuritis and anterior ischemic optic neuropathy: distinctive features. Graefes Arch Clin Exp Ophthalmol. 1998;236(3):188-192.

2. Nakajima H, Hosokawa T, Sugino M, Kimura F, Sugasawa J, Hanafusa T, Takahashi T. Visual field defects of optic neuritis in neuromyelitis optica compared with multiple sclerosis. BMC Neurol. 2010;10:45.

3. Foroozan R, Buono LM, Savino PJ, Sergott RC. Acute demyelinating optic neuritis. Curr Opin Ophthalmol. 2002;13(6):375-380 .

4. Hoorbakht H, Bagherkashi F. Optic neuritis, its differential diagnosis and management. Open Ophthalmol J. 2012;6:65-72.

5. Hayreh SS. The blood supply of the optic nerve head and the evaluation of it - myth and reality. Prog Retin Eye Res. 2001;20(5):563-593.

6. Hayreh SS. Ischemic optic neuropathy. Prog Retin Eye Res. 2009;28(1):34-62. 